Les intendants au cœur de la crise de l'Ancien Régime : 1783-1791. Les généralités d'Alençon, Caen, Rouen, Rennes, Orléans, Bourges, Moulins, Poitiers, Limoges, Tours, Riom et Dijon

\title{
Alain Cohen
}

\author{
(2) OpenEdition \\ Journals \\ Édition électronique \\ URL : https://journals.openedition.org/ahrf/11880 \\ DOI : 10.4000/ahrf.11880 \\ ISSN : 1952-403X \\ Éditeur : \\ Armand Colin, Société des études robespierristes \\ Édition imprimée \\ Date de publication : 1 décembre 2010 \\ Pagination : 101-110 \\ ISBN : 978-2-200-92634-2 \\ ISSN : 0003-4436
}

Référence électronique

Alain Cohen, «Les intendants au cœur de la crise de l'Ancien Régime : 1783-1791. Les généralités d'Alençon, Caen, Rouen, Rennes, Orléans, Bourges, Moulins, Poitiers, Limoges, Tours, Riom et Dijon », Annales historiques de la Révolution française [En ligne], 362 I octobre-décembre 2010, mis en ligne le 01 décembre 2013, consulté le 23 avril 2022. URL : http://journals.openedition.org/ahrf/11880 ; DOI : https://doi.org/10.4000/ahrf.11880 


\section{LES INTENDANTS AU COEUR DE LA CRISE DE L'ANCIEN RÉGIME : 1783-1791. \\ Les généralités d'Alençon, Caen, Rouen, Rennes, Orléans, Bourges, Moulins, Poitiers, Limoges, Tours, Riom et Dijon.}

Alain COHEN

La crise de l'Ancien Régime est un thème récurrent de l'histoire du dernier quart $d u x{ }^{e}{ }^{e}$ siècle. Un très récent débat consacré aux origines financières de la Révolution française ${ }^{1}$ a permis de revenir sur cette crise de l'Ancien Régime. Or, cette dernière fut, en grande partie, liée au sort des intendants, institution essentielle de la monarchie française. Le statut et les fonctions des intendants ont fait l'objet de récentes recherches conduites par des historiens, tels que Colette Brossault, Maïté Etchechoury, Cédric Glineur, René Grevet. Les intendants sont, en effet, au cœur d'un programme de publications scientifiques du CTHS consacré au Mémoire pour l'instruction du duc de Bourgogne, dont les éditions critiques, amorcées depuis 1977, se poursuivent ${ }^{2}$.

Toutefois, la fin des intendants n'a pas été traitée dans l'historiographie, même la plus récente, à l'exception de quelques travaux portant

(1) «Retour sur les origines financières de la Révolution française », Regards croisés avec la participation de Marie-Laure Legay, Joël Félix, Eugène White, Annales Historiques de la Révolution française, $\mathrm{n}^{\circ} 356$, avril/juin 2009, pp. 183-201.

(2) Voir L'intendance du Dauphiné en 1698, Mémoire pour l'instruction du duc de Bourgogne, édition critique par Bernard Bonnin et René Favier, Paris, Éditions du CTHS, 2005. Voir liste des éditions critiques dans la bibliographie établie par Michel BIARD, Les lilliputiens de la centralisation. Des intendants aux préfets : les hésitations d'un "modèle français », Seyssel, Champ Vallon, 2007. 
sur trois intendances ${ }^{3}$. Les raisons de cette quasi-absence de travaux sur la fin des intendants méritent d'être expliquées. Sans doute, les historiens ne sont guère enclins à étudier la fin peu glorieuse ou peu exaltante d'une institution vouée à disparaître concomitamment au déclin et à la fin de la monarchie. Pourtant, la fin des intendants offre de vastes possibilités de recherche d'un grand intérêt parce qu'elles permettent de comprendre le sens de l'évolution de cette institution entre le $\mathrm{XVII}^{\mathrm{e}}$ et le $\mathrm{XVIII}^{\mathrm{e}}$ siècles, étudiée, tout particulièrement, depuis une trentaine d'années notamment par des historiens comme François Olivier-Martin, Maurice Bordes, François-Xavier Emmanuelli et bien d'autres qui ne peuvent tous être cités dans ce texte.

La fin des intendants soulève plusieurs problématiques liées au bilan même de leur action. L'extension de leur rôle de magistrats financiers à celui d'administrateurs pouvait-elle contribuer à l'évolution de la monarchie afin de lui permettre de répondre aux défis du dernier quart du $\mathrm{XVIII}^{\mathrm{e}}$ siècle? Autre problématique : quelle a été réellement l'efficacité des intendants, alors que les critères de mesure de cet aspect divisent encore les historiens, comme Maurice Bordes, Roland Mousnier ou FrançoisXavier Emmanuelli? Les critiques adressées aux intendants durant les $\mathrm{XVII}^{\mathrm{e}}$ et $\mathrm{XVIII}{ }^{\mathrm{e}}$ siècles ont miné leur autorité et, en définitive, leur efficience, alors que du point de vue de la technique juridique, certains historiens du droit, comme François Saint-Bonnet et Yves Sassier ${ }^{4}$, soutiennent que : "L'intendant, en qualité de juge, introduit des techniques et des comportements radicalement nouveaux $\rangle^{5}$. On peut d'ailleurs ajouter, dans le même sens, que les intendants furent les fondateurs d'un ordre administratif lorsque la notion d'intérêt public apparut dans un certain nombre d'actes juridiques à partir de l'édit du 21 février 1641 dit de Saint-Germain-en-Laye.

L'analyse de ces problématiques et des solutions qui en découlent rend nécessaire des recherches juridiques à l'échelon national, comme le

(3) René GREVET, « La fin des intendances et la transition administrative dans les provinces septentrionales (1789-1790) », Intendants et préfets dans le Nord et Pas-de-Calais (XVII-XX $X^{e}$ siècle), Actes du colloque d'Arras de mars 2000, dir. A. LotTin et A. CRÉPIN, Arras, Presses Université, 2002. Voir aussi Henri Fréville qui donne des explications très complètes sur la fin de l'intendance de Bretagne, même si sa thèse (publiée) porte sur l'histoire de l'intendance de Bretagne depuis sa création, L'Intendance de Bretagne (1689-1790). Essai sur l'histoire d'une intendance en pays d'États au XVIII ${ }^{e}$ siècle, Rennes, Plihon, 1953, 3 vol.

(4) François Saint-Bonnet, Yves SAssier, Histoire des institutions avant 1789, Paris, Montchrestien, 2004.

(5) Ibid., p. 377 
soulignait déjà François-Xavier Emmanuelli ${ }^{6}$. Pour répondre à ce besoin, les recherches conduites dans le cadre de cette thèse ${ }^{7} l^{1}$ 'ont été, pour une large part, sous l'angle de l'histoire des institutions publiques, sans négliger pour autant les aspects politiques, voire économiques de la période traitée. Eu égard au nombre des intendances (32 ou 33), il était pratiquement impossible, pour un seul doctorant, de toutes les analyser afin de décrire et expliquer la fin des intendants. Il a donc fallu procéder à des choix géographiques et, bien sûr, tenir compte des travaux déjà réalisés. Une grande partie de l'Ouest (Normandie et Bretagne), le Centre (généralités d'Orléans, Bourges, Poitiers, Tours, Limoges, Moulins, Riom) et la Bourgogne (pays d'États) ont été retenus, soit 12 intendances (plus du tiers de l'ensemble des intendances). Les intendances de Caen et de Tours ont bien été étudiées. Mais, il fallait poursuivre les recherches audelà de 1783 pour prendre en compte la fin des intendances et notamment l'année 1787 (généralisation des assemblées provinciales dans les pays d'élection). Les autres pays d'élection du Centre, de l'Ouest et du Massif Central n'ont pas été analysés du point de vue de la fin des intendances, alors que les archives départementales offrent des ressources très intéressantes. Les intendances de Paris, Lyon, Franche-Comté, de Bordeaux, d'Alsace, de Troyes, d'Aix-en-Provence ont déjà fait l'objet de travaux remarquables qui ne nécessitaient pas de nouvelles recherches. En revanche, la fin des intendances de Bretagne et de Bourgogne méritait d'être examinée tout particulièrement en raison de leur statut commun de pays d'États (par opposition aux pays d'élection rappelés plus haut). Au total, 12 intendances ont été étudiées dans cette thèse (soit plus du tiers de leur nombre total).

Pour mener à bien ces recherches, le recours aux Archives nationales était indispensable notamment pour retrouver les pièces des dossiers judiciaires des intendants poursuivis devant le Tribunal révolutionnaire. Les Archives nationales ont livré également de très nombreuses correspondances échangées entre les intendants et les contrôleurs généraux des finances contenant d'intéressants documents concernant les relations des intendants avec les assemblées provinciales, les États, les cours

(6) François-Xavier Emmanueldi, Un mythe de l'absolutisme bourbonien : l'Intendance du milieu du XVII siècle à la fin du XVII ${ }^{e}$ siècle (France, Espagne, Amérique), Aix-en-Provence, Publications de l'Université de Provence-Honoré Champion, 1981, p. 177.

(7) Thèse soutenue à l'Université de Rouen le 27 octobre 2009. Jury composé de Mme Christine Le Bozec (présidente), M. Michel Biard (directeur), M. Hervé Leuwers, professeur à l'Université de Lille III, M. Claude Michaud, professeur émérite à l'Université de Paris I. 
souveraines, les parlements et le personnel des intendances. Mais, ces recherches devaient être conduites en fonction de choix méthodologiques précis. Il fallait, en effet, recouper les pièces livrées par les Archives nationales avec les documents émanant d'autres fonds d'archives, comme ceux de l'Assistance publique (fonds privés de l'ancien intendant Auget de Montyon) ou des fonds d'archives des départements correspondant à tout ou partie des intendances délimitées dans le corpus de cette thèse. Cette orientation fut à l'origine de recherches dans une douzaine de fonds d'archives départementales. Les thèmes, qui ont fait l'objet de dépouillements dans ces fonds, portaient sur les relations entre les intendants, d'une part, et les assemblées provinciales, États, parlements d'autre part; également entre les intendants et les nouvelles administrations prévues par le décret du 22 décembre 1789 et enfin les relations sociales au sein des intendances jusqu'à leur totale extinction.

Ces recherches archivistiques ne pouvaient épuiser les débats sur les fonctions et le rôle des intendants dans la mesure où l'étude de la personnalité et du destin de chacun d'entre eux aidait à comprendre l'évolution variable d'une intendance à une autre. D'où le recours au travail prosopographique qui a porté sur les intendants les plus emblématiques et à propos desquels sont disponibles des ouvrages et des biographies comme Auget de Montyon (Auvergne et La Rochelle), Sénac de Meilhan (Valenciennes), Dufaure de Rochefort (Rennes), Amelot de Chaillou (Dijon), Bertrand de Molleville (Rennes), Boula de Nanteuil (Poitou). Ces biographies ont permis de compléter la description des fonctions des intendants par les apports fournis par l'histoire de chaque intendance aux relations entre les corps intermédiaires et le personnel de cette institution.

Le travail réalisé dans le cadre de cette thèse s'articule autour de trois axes majeurs (de 3 à 6 chapitres par partie), selon un plan chronologique retraçant les étapes du déclin des intendants jusqu'à leur disparition administrative et/ou physique. Dans le cadre de la première partie consacrée aux causes du déclin des intendants, il fallait établir un bilan historiographique en distinguant certains auteurs contemporains de la fin des intendants (Auget de Montyon et Sénac de Meilhan, également intendants $\mathrm{du} \mathrm{XVIII}^{\mathrm{e}}$ siècle; ou ayant étudié avec grand talent les intendants, comme Tocqueville, auteur d'ouvrages historiques parus entre 1835 et 1856 ) et les historiens contemporains (fin XIX ${ }^{\mathrm{e}}$, comme Albert Babeau, ou au titre des années 1920-1980: Paul Ardascheff, Maurice Bordes, François-Xavier Emmanuelli et Roland Mousnier). De la confrontation entre ces auteurs, sont apparus à la fois l'ambiguïté du rôle des intendants et le caractère très contesté du bilan de leur action dans l'histoire de cette 
fin de la monarchie. Le malaise exprimé par ces auteurs est à mettre en rapport avec la fin calamiteuse de cet Ancien Régime qui n'a pu répondre aux multiples défis qu'il devait affronter. Et pourtant, plusieurs historiens ont souligné les aspects positifs de cette institution des intendants, notamment leur rôle précurseur et novateur pour le développement de l'action de l'État annonçant, par certains côtés, l'institution des préfets en vigueur à partir de l'année 1800 .

Néanmoins, le déclin des intendants a été aussi accéléré par les vives critiques adressées dès le début $\mathrm{du}$ XVIII ${ }^{\mathrm{e}}$ siècle par des écrivains, comme Fénelon et Saint-Simon, un ministre, comme Necker, ou un publiciste, comme Brissot de Warville dans le dernier quart du siècle. Les trois chapitres suivants de cette première partie illustrent les conflits opposant les intendants aux assemblées provinciales (dans les pays d'élection) et aux États de Bourgogne et de Bretagne, aux parlements et cours des Aides. Ces dernières passes d'armes constituèrent les derniers feux de cette lutte des intendants pour la défense de leurs fonctions, de leur statut et de leur rôle politique majeur. Le dernier chapitre de la première partie relatif aux critiques développées par les cahiers de doléances et la presse administre la preuve que la défense pro domo des intendants n'avait aucune chance d'être admise en raison du poids de l'opinion publique annonciatrice d'un nouvel ordre constitutionnel rejetant en bloc les fins et moyens de l'Ancien Régime.

La seconde partie relative à la transmission des pouvoirs pendant les années 1790-1791, répartie en 4 chapitres, coïncide précisément avec le début de la Révolution, qui, en jetant les bases d'une nouvelle organisation des pouvoirs publics, scelle en même temps la disparition administrative des intendants (décrets des 14 et 22 décembre 1789). Les nombreux textes analysés dans cette thèse démontrent que la mise en place des nouvelles structures administratives soulevait de nombreux problèmes techniques et politiques, alors que les pouvoirs (législatif et exécutif) devaient assurer la transition entre deux systèmes institutionnels et donc maintenir, au moins pendant un certain temps, certains organes d'Ancien Régime, comme les intendants et officiers municipaux. La circulaire du 25 juin $1790^{8}$ du contrôleur général des finances devait normalement contribuer

(8) AN, H1 621, pièce 428. Le Contrôleur général des finances ne fut pas le seul ministre à veiller au bon déroulement de la transmission des archives détenues par les intendants. Le garde des Sceaux adresse aussi le 26 juin 1790 une instruction à l'intendant de Caen (Cordier de Launay) qui est plus précise que celle du contrôleur général, en raison du contenu des documents intéressant la Guerre, la Marine, la police des Étapes, le service de la Maréchaussée et le casernement des Brigades. 
à la passation des pouvoirs entre les deux administrations (sortante et entrante). Toutefois, les arrières pensées et craintes d'une exploitation à des fins politiques de certaines correspondances ministérielles constituaient des éléments de nature à expurger, voire à détruire, un certain nombre de pièces utiles aux administrations départementales.

Les textes administratifs adoptés ne pouvaient garantir, par euxmêmes, la régularité des opérations de transmission entre administrations (intendances et directoires de départements). Tout dépendait, en réalité, de l'entente sur le terrain entre le personnel des bureaux des intendances (commis, chefs de bureaux et subdélégués) et les services rattachés aux directoires des départements. Les nombreuses sources disponibles permettent de constater une assez bonne transmission des archives entre administrations, comme le prouvent les registres des délibérations de plusieurs départements ou les Adresses de la Commission de liquidation des anciennes affaires de la Bretagne ${ }^{9}$. Et pourtant, les circonscriptions des généralités ne correspondaient en aucune manière aux limites des départements et districts. Les généralités, dont le périmètre était très étendu, donnèrent naissance généralement à plusieurs départements, ce qui ne facilitait pas la transmission des archives. Il faut aussi ajouter que la bureaucratie royale était particulièrement étoffée puisqu'elle reposait sur des règles très précises de formalisme administratif et comptable. D'où l'énormité de la masse des pièces à transmettre aux nouvelles administrations désireuses, par ailleurs, de les rassembler en totalité. Cette conception pouvait paraître excessive alors que les problèmes essentiels ne se situaient pas au plan du transfert intégral des archives, mais plutôt au niveau de la priorité à donner à la reprise des rôles d'imposition qui était essentielle à la continuité des rentrées fiscales. Les difficultés de transmission des pouvoirs et donc des archives expliquèrent les retards importants constatés dans le recouvrement des impôts directs et indirects, phénomène aggravé par une rébellion fiscale en Berry et Bretagne. D'où la mesure prise par l'Assemblée nationale de proroger les compétences fiscales des commissions intermédiaires (émanation des assemblées provinciales) jusqu'au 31 décembre $1790^{10}$.

Un autre aspect administratif, techniquement délicat sur un plan pratique, résidait dans l'incertitude quant à la date du transfert effectif

(9) Adresse du 6 novembre 1790 de la Commission de liquidation des anciennes affaires de la Bretagne à l'Assemblée nationale, AD Ille-et-Vilaine, C1449.

(10) Marie-Laure Legay, « La fin du pouvoir provincial (4 août 1789-21 septembre 1791) », Annales historiques de la Révolution française, $\mathrm{n}^{\circ}$ 332, avril-juin 2003, p. 24-53. 
des pouvoirs entre administrations (sortante et entrante). Ce point était très important parce qu'il conditionnait la transmission des documents fiscaux (notamment l'assiette et la perception des impositions). Le décret du 22 décembre 1789 (art. 9, $3^{\mathrm{e}}$ section) précisait que les intendants devaient cesser leurs fonctions « aussitôt que les administrations de département seront entrées en fonction ». Le critère retenu fut la date de clôture de la $1^{1 \text { ère }}$ assemblée nommant les membres du directoire du département ${ }^{11}$. Par ailleurs, grâce à l'esprit de coopération des intendants et de leurs adjoints (subdélégués, chefs de bureaux et commis), la transmission s'effectua généralement dans les meilleures conditions. Mais, on doit signaler une exception notable avec le cas particulier de la Bourgogne qui connut des relations très conflictuelles entre les Élus (représentants des États du même nom) et les directoires des départements de la Côte-d'Or et de la Saône-et-Loire. En effet, en Bourgogne, le rôle de l'intendant était effacé.

La transmission des pouvoirs entre intendants et directoires des départements ne pouvait s'effectuer sans un délai suffisant de transition. En effet, jusqu'à la mise en place effective des nouvelles administrations, les intendants devaient continuer à assurer le maintien de l'ordre et veiller à l'approvisionnement en vivres des populations de la généralité. Or, l'accomplissement de ces fonctions était perturbé par les circonstances qui désorganisaient le fonctionnement des marchés de céréales (émeutes contre les hausses du prix du pain, saisies des convois). Grâce aux archives privées de l'intendant de Tours ${ }^{12}$, d'Aine, il a été possible de retracer les difficultés auxquelles dut faire face cet intendant lorsque les prix des grains dérapèrent, provoquant ainsi de graves atteintes à l'ordre public (marsavril 1789). Cette détérioration de la situation économique et sociale devait entraîner des démissions en cascade (celle de la municipalité de Tours et celle de l'intendant en juillet-août 1789). De tels événements ne pouvaient perdurer sans altérer gravement le fonctionnement de l'État qui dépendait étroitement de la continuité des services administratifs (en l'occurrence les intendances). D'où l'examen des conséquences de la suppression des intendances, objet de la troisième partie.

La troisième partie est subdivisée en trois chapitres : le sort du personnel des intendances et sa défense présentés par les intendants; ces derniers, proprement dits, cibles du nouveau pouvoir central et les

(11) Lettre du Contrôleur général Lambert du 2 juillet 1790 adressée à l'intendant de Limoges, Meulan d'Ablois, A.D. Haute-Vienne, C1.

(12) AN, $291 \mathrm{AP} / 1$. 
hôtels d'intendance. Le sort du personnel des intendances a pu être étudié de manière très précise grâce aux correspondances entre intendants et contrôleurs généraux. Ainsi, les dossiers de plusieurs collaborateurs de l'intendant Amelot de Chaillou (Dijon) permettent de reconstituer plusieurs litiges sociaux et de décrire l'amorce d'un statut du personnel des intendances. Ces aspects de la gestion du personnel ont été complétés par le recensement des actions développées par les intendants pour assurer le reclassement de leur personnel après la suppression des intendances. Ainsi, est-on en présence d'une véritable radiographie très instructive de la carrière, par exemple, de chaque collaborateur de l'intendance de Bretagne $^{13}$ et des perspectives de son reclassement éventuel. Mais, si le personnel des intendances a dans l'ensemble pu être reclassé dans d'assez bonnes conditions (à l'exception, bien sûr, des commis ou employés admis à faire valoir leur droit à la retraite), la situation personnelle et professionnelle des intendants allait connaître un dénouement beaucoup plus difficile, voire pour certains tout à fait dramatique.

Le détonateur de la détérioration de la situation des intendants fut matérialisé par l'assassinat de celui de Paris (Bertier de Sauvigny), intervenu le 22 juillet 1789 . Ce fut, en effet, le signal d'un sauvequi-peut général qui conduisit de nombreux intendants à donner leur démission, accélérant la désorganisation des intendances et le bon déroulement des opérations de transmission des archives. Mais, le pire était encore à venir dans la mesure où une quinzaine d'intendants firent l'objet de poursuites pénales, notamment devant le Tribunal révolutionnaire ${ }^{14}$. D'après les minutes des procès indiqués en note ci-dessous, le passé de ces intendants n'a pas été l'élément clé de la décision de condamnation à la peine capitale, mais plutôt une cause aggravante de l'incrimination principale (comme émigré). Les autres procès engagés à l'encontre d'autres intendants, assignés dans le cadre de groupes de prévenus de complots, confirment cette tendance du Tribunal révolutionnaire à ne pas retenir la qualité d'ancien intendant, comme motif principal de poursuite pénale ${ }^{15}$. Au total, une proportion assez forte d'intendants $(60 \%)$ a été condamnée à mort par rapport au nombre total d'intendants poursuivis

(13) Mémoire pour les commis des bureaux de l'intendance de Bretagne, ibid., H1 589, pièce 76 .

(14) Voir dossiers judiciaires de Maussion (intendant de Rouen), ibid., W 332 et Guéaux de Reverseaux (intendant de Moulins et de La Rochelle), ibid., W 323.

(15) Voir article de A. Cohen, «Les procès des anciens intendants durant la Révolution », Annales historiques de la Révolution française, avril-juin 2009, nº 356, p. 29-56. 
La conclusion de cette thèse insiste sur l'œuvre importante réalisée par les intendants, sur l'innovation des méthodes d'administration de la justice rendue par ces mêmes intendants et enfin sur leur rôle positif comme administrateurs. Le concert de dures critiques qu'ils subirent à partir du $\mathrm{XVII}^{\mathrm{e}}$ siècle, et pendant une grande partie du siècle suivant, ne peut que susciter l'étonnement parce qu'ils ont été les boucs émissaires de l'Ancien Régime, livrés par le roi à la vindicte des officiers de finances, des cours souveraines, des assemblées provinciales, des États qui voyaient dans les intendants les instruments du despotisme ministériel et aussi l'incarnation des iniquités du système fiscal. Les cahiers de doléances ont relayé ces critiques des corps constitués, ce qui contribua à aggraver le discrédit subi par les intendants. À partir de juillet 1789, l'autorité du roi était de plus en plus chancelante. Or, pour faire face à la montée des périls, le roi aurait dû s'appuyer sur ces relais que constituaient les intendants, au moins pour quelque temps. Il faut bien dire que le chef de l'exécutif avait d'autres préoccupations en raison du conflit violent qui l'opposait à partir d'octobre 1789, et encore davantage pendant deux longues années, à la représentation nationale et aux partis qui la soutenaient. En tout état de cause, même en faisant abstraction de ces considérations éminemment politiques, le roi ne pouvait même plus faire appel aux intendants puisqu'il les avait purement et simplement abandonnés depuis le début des années 1780 et tout particulièrement lorsqu'il voulut imposer en 1787 ces solutions de replâtrage des institutions publiques reposant sur des assemblées provinciales censées résoudre la crise des finances publiques et offrir à la monarchie finissante un répit en sollicitant le consentement des propriétaires au redressement des finances publiques. Mais, ces solutions arrivaient trop tard et ne firent qu'aggraver la crise fiscale et financière d'autant plus que le crédit (financier) de la monarchie était au plus bas, selon les cotations du marché financier ${ }^{16}$.

Alain CoHEN

(16) Voir à ce sujet les analyses d'Eugene White dans les Regards croisés, « Retour sur les origines financières [...] », op. cit., Annales historiques de la Révolution française, $\mathrm{n}^{\circ} 356$, avril-juin 2009, p. 197. Voir note (23) de l'article précité. 